\title{
Reflets
}

Revue d'intervention sociale et communautaire

\section{Violences faites aux femmes et contextes minoritaires}

\section{Isabelle Côté, Simon Lapierre et Joscelyne Levesque}

Volume 21, numéro 1, printemps 2015

Violences faites aux femmes et contextes minoritaires

URI : https://id.erudit.org/iderudit/1032546ar

DOI : https://doi.org/10.7202/1032546ar

Aller au sommaire du numéro

Éditeur(s)

Reflets, Revue d'intervention sociale et communautaire

ISSN

1203-4576 (imprimé)

1712-8498 (numérique)

Découvrir la revue

Citer ce document

Côté, I., Lapierre, S. \& Levesque, J. (2015). Violences faites aux femmes et

contextes minoritaires. Reflets, 21(1), 10-15. https://doi.org/10.7202/1032546ar d'utilisation que vous pouvez consulter en ligne.

https://apropos.erudit.org/fr/usagers/politique-dutilisation/ 


\section{Violences faites aux femmes et contextes minoritaires}

\section{Isabelle Côté}

Doctorante, École de service social, Université de Montréal

Simon Lapierre, Ph. D.

Professeur agrégé, École de service social, Université d'Ottawa

Joscelyne Levesque, MSS

Coordonnatrice de la formation pratique et des stages

École de service social, Université d'Ottawa

\section{Violences faites aux femmes}

Depuis les années 1970, des militantes et chercheuses féministes travaillent de concert pour faire reconnaitre les violences faites aux femmes comme important problème social, tant dans les communautés locales que sur les scènes nationale et internationale (Schechter, 1982; Dobash et Dobash, 1992). En se basant sur les expériences individuelles et collectives des femmes, elles dénoncent ces violences et les définissent comme une manifestation du patriarcat, puisqu'elles émanent des inégalités et des rapports de pouvoir entre les femmes et les hommes. Militantes et chercheuses ont collaboré progressivement à la mise en place d'un vaste réseau de ressources visant à assurer la sécurité et à soutenir les femmes victimes de violences, incluant les maisons d'hébergement pour femmes victimes de violence conjugale, les centres d'aide et de lutte contre les agressions à caractère sexuel, et les centres de femmes (Coderre et Hart, 2003; Prud'homme, 2010). 
Malgré une reconnaissance accrue du phénomène et sa prise en charge par des actrices et des acteurs politiques, communautaires et institutionnels, un nombre considérable de femmes, ici et ailleurs dans le monde, sont toujours la cible de la violence des hommes. Au Canada, les données policières révèlent qu'environ 173600 femmes de 15 ans et plus ont été victimes d'un crime violent au cours de l'année 2011 (Statistique Canada, 2013). Les actes de violence les plus souvent rapportés sont les voies de fait (59 \%), les menaces (13\%), les agressions sexuelles $(7 \%)$ et le harcèlement criminel $(7 \%)$. Les hommes, souvent le partenaire intime (45\%) ou des amis ou connaissances de la femme (27\%), étaient responsables de $83 \%$ de ces actes. Par ailleurs, comme la majorité des incidents de violence ne sont pas signalés aux services policiers, les taux réels de victimisation des femmes sont beaucoup plus élevés que ceux-là.

\section{Contextes minoritaires}

Même si ces violences sont présentes dans toutes les sphères de la société, force est de constater que leurs manifestations et leurs conséquences peuvent être différentes selon le contexte social, politique, culturel et économique dans lequel elles s'inscrivent. En effet, les femmes vivant en situation minoritaire, en raison de leur langue, de leur âge, de leur situation de handicap, de leur orientation sexuelle, de leur religion ou de leur origine ethnique, peuvent connaître des expériences différentes et être confrontées à certaines barrières qui accentuent leur vulnérabilité face à la violence. À cet égard, notons l'importante contribution de femmes racisées qui, en s'appuyant sur leur vécu, ont démontré que leur expérience de victimisation était très différente de celle décrite par des féministes blanches (Crenshaw, 1991; Richie, 1996). Leur oppression résultait du patriarcat, mais aussi d'un système raciste soutenu à la fois par les hommes et les femmes de race blanche.

De plus, les femmes peuvent aussi avoir un accès plus limité aux ressources d'aide ou encore être confrontées à des pratiques 
qui ne tiennent pas compte de leurs réalités particulières.Toutefois, malgré des lacunes et des défis importants, des efforts ont été déployés afin de développer des pratiques mieux adaptées aux diverses réalités des femmes victimes de violence, incluant des ressources "par et pour " les femmes vivant dans des contextes minoritaires. Par exemple, une recherche récente démontre clairement que le manque de disponibilité et d'accès aux services en français pour les femmes et les enfants francophones vivant dans un contexte de violence conjugale en Ontario risque de compromettre leur sécurité, leur santé et leur bien-être (Lapierre, et collab., 2014).

Au Canada, de nombreux rapports ont mis en lumière la réalité particulière des femmes autochtones, dont plusieurs ont été assassinées ou portées disparues (Feinstein et Pearce, 2015; GRC, 2014). Ces rapports ont aussi révélé des pratiques policières préoccupantes, voire totalement inadéquates. Malgré ces constats, le Gouvernement du Canada persiste dans son refus de mettre en place une enquête publique sur la situation de ces femmes assassinées ou disparues, ce qui dénote une attitude colonialiste envers les communautés autochtones.

\section{Contenu et structure du numéro}

Dans ce numéro de Reflets, nous réunissons des textes sur les violences faites aux femmes vivant en contextes minoritaires, dans l'objectif de contribuer à l'avancement des connaissances dans ce domaine et d'améliorer les politiques sociales et les pratiques d'intervention auprès de ces femmes. Le numéro s'amorce avec une entrevue accordée par une Autochtone qui témoigne des réalités vécues au Canada par les femmes autochtones. Ses propos permettent de constater les impacts directs, importants et continus du colonialisme et du patriarcat sur leurs parcours de vie.

Cinq articles composent notre Dossier, abordant différentes dimensions du phénomène des violences faites aux femmes en contextes minoritaires. Les trois premiers portent sur les violences 
faites aux femmes francophones en situation minoritaire en Ontario. Dans le premier article, Linda Cardinal, Marie-Luce Garceau et Ghislaine Sirois examinent de manière exploratoire le rôle des intervenantes du secteur de la violence faite aux femmes dans le développement des services en français en Ontario. Débutant avec un rappel historique des recherches et des actions réalisées au cours des vingt dernières années, les auteures soulignent que peu d'études ont porté sur les savoirs expérientiels des intervenantes dans le secteur de la violence faite aux femmes. Les données qu'elles ont recueillies ont permis de mettre en évidence le rôle de ces intervenantes dans le développement des services en français dans la province.

Dans le deuxième article, Laurence Clennett-Sirois soulève les défis auxquels font face les jeunes femmes monoparentales victimes de violence conjugale lorsqu'elles vivent en milieu semi-rural dans un contexte francophone minoritaire. S'inscrivant dans une perspective écologique, cette recherche-action a permis d'identifier des "facteurs personnels de vulnérabilité " et des "facteurs associés à l'environnement " qui accentuent les impacts des violences vécues par ces jeunes femmes et qui rendent plus difficile la sortie de la relation avec un conjoint violent. L'auteure propose de mettre en place une ressource régionale pour les jeunes femmes monoparentales, facilitant ainsi leur accès au logement sécuritaire et abordable, aux services de garde et aux services soutenant leur développement personnel, académique et professionnel.

Un troisième article examine les enjeux liés à la prostitution et aux services en droit de la famille de manière à illustrer le développement des connaissances dans le domaine de violence faite aux femmes en Ontario français. Marie-Luce Garceau, Ghislaine Sirois et Marc Charron portent une attention particulière aux mécanismes de concertation qui nourrissent les pratiques d'intervention et mènent à l'action.

Un quatrième article, signé Dominique Damant, Jeanne Bernard, Marianne Chbat et Catherine Flynn, aborde aussi la question de la langue en se penchant sur la situation de femmes anglophones et sur l'oppression qu'elles peuvent subir 
au sein des services francophones au Québec. S'inspirant d'une analyse féministe intersectionnelle, les auteures soutiennent que l'interaction entre différents systèmes d'oppression augmente la difficulté de ces femmes à s'intégrer dans la société québécoise, accroit leur dépendance envers leur conjoint et menace leur sécurité et celle de leurs enfants.

Le cinquième article du Dossier porte sur l'expérience de femmes arabes et musulmanes victimes de violence conjugale en contexte migratoire, plus spécifiquement en lien avec leurs stratégies de mobilisation des ressources. Employant la méthode des récits de vie, Bouchra Taibi donne la parole à quatre femmes d'origine magrébine qui, loin d'être des victimes passives, ont su mobiliser de multiples ressources (formelles et informelles) pour faire face à leur situation. Les résultats de la recherche sont un appel à élargir la conception des expériences des femmes victimes de violence conjugale, en tenant compte des violences subies dans les sphères publiques et privées.

La rubrique Des pratiques à notre image présente des pratiques novatrices mises en place par quatre organismes communautaires œuvrant dans le secteur des violences faites aux femmes. D'abord, Action ontarienne contre la violence faite aux femmes (AOcVF) présente le Centre de services juridiques, une initiative qui s'inscrit dans sa lutte pour un meilleur accès à la justice pour les femmes francophones victimes de violence. Puis, Véronique Paradis et Isabelle Côté décrivent le processus entrepris par l'équipe de la Maison Unies-Vers-Femmes, lequel vise à remettre les valeurs féministes au centre de leur travail quotidien. Ensuite, Sarah Cloutier présente la Campagne du consentement, une campagne de sensibilisation mise en place par les EssentiElles, une association de femmes franco-yukonnaises. Finalement, Michèle Diotte présente un programme de prévention des agressions sexuelles développé par le CALAS de l'Outaouais; ce programme cible les personnes ayant une déficience intellectuelle. 


\section{Bibliographie}

CODERRE, Cécile, et Joanne HART (2003). "Pratiques d'intervention féministe auprès des femmes survivantes d'agression à caractère sexuel : le contexte franco-ontarien ", Reflets, revue d'intervention sociale et communautaire, Vol. 9, No 1, p. 186-210.

CRENSHAW, Kimberlé (1991). "Mapping the margins: intersectionality, identify politics, and violence against women of color ", Stanford Law Review, Vol. 43, № 6, p. 1241-1299.

DOBASH, Rebecca E., et Russell P. DOBASH (1992). Women, Violence and Social Change. Routledge, 382 pages.

FEINSTEIN, Pippa, et Megan PEARCE (2015).Violence against indigenous women and girls in Canada: review of reports and recommendations, Executive summary.

GENDARMERIE ROYALE DU CANADA (2014). Les femmes autochtones disparues et assassinées : un aperçu opérationnel national, [Rapport officiel], 23 pages.

LAPIERRE, Simon, Cécile CODERRE, Isabelle CÔTÉ, Marie-Luce GARCEAU et Chantal BOURASSA (2014). "Quand le manque de services en français revictimise les femmes victimes de violence conjugale et leurs enfants : impacts des lacunes dans l'accès aux services en français en Ontario et au Nouveau-Brunswick ", Reflets, revue d'intervention sociale et communautaire, Vol. $20, N^{\circ} 2$, p. 22-51.

PRUD'HOMME, Diane (2010). «L'intervention féministe en maison d'hébergement : une vigilance de tous les instants! », dans Christine Corbeil et Isabelle Marchand (dirs.), L'intervention féministe d'hier à aujourd'hui : portrait d'une pratique sociale diversifiée, Montréal, Les éditions du remueménage, p. 131-147.

RICHIE, Beth (1996). Compelled to crime: The gender entrapment of battered Black women, Routledge, 200 pages.

SCHECHTER, Susan (1982). Women and Male Violence:The Visions and Struggles of the Battered Women's Movement, South End Press, 378 pages.

STATISTIQUE CANADA (2013). Mesure de la violence faite aux femmes : tendances statistiques, 130 pages. 